\title{
Selecting Implications in Fuzzy Abductive Problems
}

\author{
Adrien Revault d'Allonnes Herman Akdag Bernadette Bouchon-Meunier
}

\begin{abstract}
Abductive reasoning is an explanatory process in which potential causes of an observation are unearthed. We have concentrated on the formal definition of fuzzy abduction as an inversion of the Generalised Modus Ponens given by Mellouli and Bouchon-Meunier. While studying this formalism we noticed that some observations could not be explained properly. Observations, in abductive reasoning, are made within the conclusion space of the considered rule. Their potential shape is therefore highly constrained by the implication operator used. We claim that, given a feasible observation and a set of rules, we can categorise the set of implications to be used. Since a given observation will match only part of the conclusions in the rule-set, we offer a categorisation of a rule system coherent with observed data.
\end{abstract}

Index Terms - Abductive reasoning, fuzzy inference, fuzzy implications, Generalised Modus Ponens

\section{INTRODUCTION}

$\mathcal{A}$ BDUCTIVE reasoning is an explanatory process in which potential causes of an observation are unearthed. In its classical - crisp - version it offers little lattitude for discovery of new knowledge. Placed in a fuzzy context, abduction can explain observations which did not, originally, exactly match the expected conclusions. Studying the effects of slight modifications through the use of linguistic modifiers was, therefore, of interest in order to describe the extent to which observations can be modified yet still explained and possibly create new knowledge.

We have concentrated on the formal definition of fuzzy abduction given by Mellouli and Bouchon-Meunier. Their approach of abduction [1], [2] aims at finding conditions on premise $A$ so that observation $B^{\prime}$ is satisfied. To do this, they choose to reverse the Generalised Modus Ponens (GMP), the fuzzy inference model, firstly because it ensures that the conditions on $A$ entail $B^{\prime}$, and secondly because it gives a mathematical expression of said conditions. Obviously, due to the large number of fuzzy implication and GMP operators at hand, one cannot consider such a task as a unique problem. Mellouli and BouchonMeunier therefore considered the different classes of fuzzy implications as described by Dubois and Prade in [3], [4]. In their works they reversed the GMP for two classes of implications: s-implications and r-implications. The results for s-implications gave an expression of $f_{A^{\prime}}(u)$ the conditionned premise's membership function. They chose not to delve into an in-depth study of this result, presumably because it offered no immediate difficulty. On the other hand, the reversal of the GMP for r-implications resulted in the definition of a 'maximal explanation' $A_{G}$ such that any explanation $A^{\prime}$ should be included in $A_{G}$. $A_{G}$ is given by:

Laboratoire d'Informatique de Paris 6

Université Pierre et Marie Curie

8 rue du capitaine Scott, 75015, Paris, France

Phone: (+33) 144-278-807, email: Adrien.Revault-d'Allonnes@lip6.fr

$$
\forall u \in U, f_{A_{G}}(u)=\inf _{v \in V} I_{T}\left(I_{T}\left(f_{A}(u), f_{B}(v)\right), f_{B^{\prime}}(v)\right)
$$

Mellouli and Bouchon-Meunier only studied a particular class of modifiers (viz. uncertain expansive modifiers) and then only using Gödel's implication. We wished to see if we could generalise their results to other types of modifiers and other implication/t-norm pairs.

Our aim was to generalise these results to other hedges and implications. We have chosen to study classical power modifiers as defined by Zadeh [5], and translation modifiers introduced by Bouchon-Meunier and Yao [6]. We finally considered the particular cases, defined from these translations, of reinforcement hedges which contract both supports and kernels (i.e. $f_{B^{\prime}}(v)=\min \left(f_{B}(v+\varepsilon), f_{B}(v-\varepsilon)\right)$ ) and their inverses which dilate them. These modifiers are semantically consistent with Zadeh's definition, yet their impact on the support and kernel of the original labels implies a shift in precision, both formally and intuitively.

Our results were incompatible with established theories. We proved, for one, that the extension of Mellouli and Bouchon-Meunier's formal results on abduction sometimes generates incoherent results. This paper will introduce a way of using this to give a semantically consistent interpretation of a rule set. We will show where this incompatibility comes from and derive from it a selection method of fuzzy implication, based on observable data.

\section{Classification With Respect to observations}

\section{A. Origin of inconsistency}

We have shown in [8] that given a gradual-rule abductive problem, Łukasiewicz's implication and an observation such that $B^{\prime} \supset B$ and $\inf _{v \in V} f_{B^{\prime}}(v)=0$ then $A_{G}=A$. Our problem is that this result is:

- Inconsistent with general results on the Generalised Modus Ponens:

- if $A^{\prime} \subseteq A$ then $B^{\prime}=B$

- if $A^{\prime} \supset A$ then $B^{\prime} \supset B$

- Inconsistent with previous results on abduction

- if $B_{1}^{\prime} \subset B_{2}^{\prime}$ then $A_{G_{1}} \subset A_{G_{2}}$, here if $B_{1}^{\prime}=B$ then $A_{G_{1}}=A$

- Inconsistent with Lukasiewicz as an $\mathrm{r}-$ and $\mathrm{s}-$ implication

- Any satisfactory explanation $A^{\prime}$ is such that $A^{\prime} \subset A_{G}$

- r-implication : $A_{G}=A$

- s-implication : $A^{\prime}=U$

Furthermore, we claim that, given a feasible observation and a set of rules, we can categorise the set of implications to be used. Since a given observation will match only part of the conclusions in the rule-set, we offer a categorisation of a rule system coherent with observed data. 
Indeed, in most cases the semantic interpretation of a rule will be given a priori, even if the rule is learnt, and an implication operator chosen regardless of its potential inconsistency with the data. Our approach aims at building entailment consistent rule-subsets, interpreting these with respect to the observed data and giving them the semantic interpretation of the corresponding implication-subset [3], $[4]$.

To do this, we need to classify the shapes which may be reached via GMP for each implication and consistent GMP-operator. This type of study has been led in the past, yet since their use was to be different the results are neither sufficiently precise nor general. Classical studies of the GMP have typically looked at what a precise observation in a given fuzzy premise will generate or at very local modifications [7]. The problem here is that we need to rule out, or accept, a given shape for an implication. So we need to extend the existing results to be certain that no unexpected case is overlooked.

\section{B. Describing GMP conclusions \\ B.1 Foreword}

Before we present our study of GMP conclusions with respect to the fuzzy subsets they entail, we think it wise to remind the reader of the general expression of the GMP conclusion and of the expressions of the fuzzy implications we will study, and their classification.

For a fuzzy rule of the type 'If $u$ is $A$ then $v$ is $B$ ' and an observation $A^{\prime}$, the expected conclusion is given by:

$$
f_{B^{\prime}}(v)=\sup _{u \in U} \top\left(f_{A^{\prime}}(u), I\left(f_{A}(u), f_{B}(v)\right)\right)
$$

Where $A$ and $A^{\prime}$ are fuzzy subsets of $U, B$ and $B^{\prime}$ fuzzy subsets of $V, I$ some fuzzy implication and $T$ an adequate (i.e. the crisp limit cases are preserved by the joint use of $I$ and $T$ ) Generalised Modus Ponens operator, or t-norm.

We will study the fuzzy implications and their respective GMP operators as given by [7] outlined in table I. The relevant GMP operators are given in table II.

TABLE II

FUZZY GMP OPERATORS

\begin{tabular}{|l|l|}
\hline Operator & Expression \\
\hline Lukasiewicz & $\mathrm{\top}(a, b)=\max (0, a+b-1)$ \\
\hline Zadeh & $\mathrm{\top}(a, b)=\min (a, b)$ \\
\hline Goguen & $\mathrm{\top}(a, b)=a \times b$ \\
\hline
\end{tabular}

\section{B.2 Reichenbach}

A rule used with Reichenbach's implication and Łukasiewicz's GMP operator will conclude on something of the form:

$$
f_{B^{\prime}}(v)=\sup _{u \in U} \max \left(0, f_{A^{\prime}}(u)+f_{A}(u) \times\left(f_{B}(v)-1\right)\right)
$$

From which we draw the following constraints on all conclusions $B^{\prime}$ :

- If $\operatorname{Kernel}\left(A^{\prime}\right) \cap \overline{\operatorname{Support}(A)} \neq \varnothing$ then $B^{\prime}=V$

- If $A^{\prime} \supseteq A$ then $B^{\prime} \supseteq B$ and $\inf _{v \in V} \overline{f_{B^{\prime}}}(v) \geqslant \sup _{u \in \overline{\operatorname{Support}(A)}} f_{A^{\prime}}(u)$

- If $A^{\prime} \subset A$ and $K \operatorname{ernel}\left(A^{\prime}\right) \cap \operatorname{Kernel}(A) \neq \varnothing$ then $B^{\prime}=B$

- Otherwise, if $A^{\prime} \subset A$ then $B^{\prime} \subset B$

\section{B.3 Willmott}

With Lukasiewicz's t-norm for GMP operator, the conclusion of a fuzzy inference given Willmott's implication is:

$$
f_{B^{\prime}}(v)=\max \left(f_{B}(v), \sup _{u \in U} f_{A^{\prime}}(u)-f_{A}(u)\right)
$$

Which gives us:

- $f_{B^{\prime}}(v) \leqslant f_{B}(v), \forall v \in V$

- $f_{B^{\prime}}(v) \geqslant \sup _{u \in U} f_{A^{\prime}}(u)-f_{A}(u), \forall v \in V$

$-\inf _{v \in V} f_{B^{\prime}}(v) \geqslant \sup _{u \in \overline{\operatorname{Support}(A)}} f_{A^{\prime}}(u)$

\section{B.4 Mamdani}

We have studied the conclusions of Mamdani rules with the min, product or Lukasiewicz GMP operators and their membership functions are:

With Zadeh's min t-norm:

$$
f_{B^{\prime}}(v)=f_{B}(v)
$$

With Goguen's product t-norm:

$$
f_{B^{\prime}}(v)=\max \left(\begin{array}{l}
\sup _{u \in U, f_{A}(u) \leqslant f_{B}(v)} f_{A^{\prime}}(u) \times f_{A}(u), \\
\sup _{u \in U, f_{A}(u) \geqslant f_{B}(v)} f_{A^{\prime}}(u) \times f_{B}(v)
\end{array}\right)
$$

With Łukasiewicz's t-norm:

$$
f_{B^{\prime}}(v)=\max \left(0, \begin{array}{l}
\sup _{u \in U, f_{A}(u) \leqslant f_{B}(v)} f_{A^{\prime}}(u)+f_{A}(u)-1, \\
\sup _{u \in U, f_{A}(u) \geqslant f_{B}(v)} f_{A^{\prime}}(u)+f_{B}(v)-1
\end{array}\right)
$$

So we have:

- $B^{\prime} \equiv B$ for Zadeh's GMP operator

- $f_{B^{\prime}}(v) \leqslant f_{B}(v), \forall v \in V$ otherwise

\section{B.5 Rescher-Gaines}

Whatever the GMP operator (min, product or Lukasiewicz's t-norm), the conclusion of a fuzzy inference given Rescher-Gaines' implication is:

$$
f_{B^{\prime}}(v)=\sup _{u \in U, f_{B}(v) \geqslant f_{A}(u)} f_{A^{\prime}}(u)
$$

And our conclusion will be such that: 
TABLE I

FuZzy implications, ClASSES AND ASSORTED GMP OPERATORS

\begin{tabular}{|l|l|l|l|}
\hline Implication & Expression & Class & Compatible t-norm(s) \\
\hline Reichenbach & $I_{R}(a, b)=1-a+a \times b$ & s-implication & Łukasiewicz \\
\hline Willmott & $I_{W}(a, b)=\max (1-a, \min (a, b))$ & Ql-implication & Łukasiewicz \\
\hline Mamdani & $I_{M}(a, b)=\min (a, b)$ & t-implication & Zadeh, Łukasiewicz, Goguen \\
\hline Rescher-Gaines & $I_{R G}(a, b)=\left\{\begin{array}{l}1 \text { if } a \leqslant b \\
0 \text { otherwise }\end{array}\right.$ & r-implication & Zadeh, Lukasiewicz, Goguen \\
\hline Kleene-Dienes & $I_{K D}(a, b)=\max (1-a, b)$ & s-implication & Łukasiewicz \\
\hline Gödel & $I_{G}(a, b)=\left\{\begin{array}{l}1 \text { if } a \leqslant b \\
b \text { otherwise }\end{array}\right.$ & r-implication & Zadeh, Lukasiewicz, Goguen \\
\hline Goguen & $I_{G n}(a, b)=\left\{\begin{array}{l}\min (b / a, 1) \text { if } a \neq 0 \\
1 \text { otherwise }\end{array}\right.$ & r-implication & Łukasiewicz, Goguen \\
\hline Lukasiewicz & $I_{L}(a, b)=\min (1-a+b, 1)$ & r- \& s-implication & Łukasiewicz \\
\hline
\end{tabular}
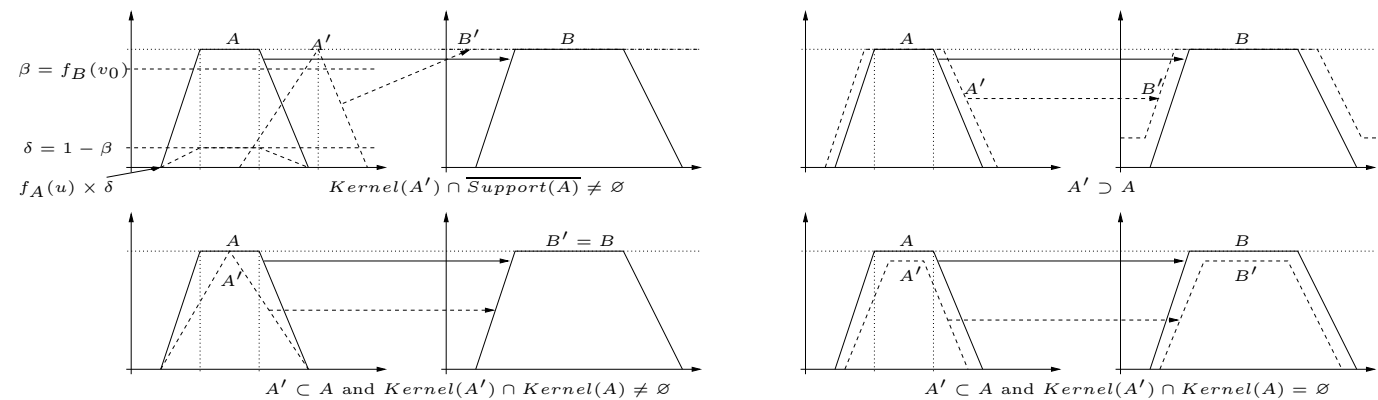

Fig. 1. GMP conclusions with Reichenbach's implication

- $\inf _{v \in V} f_{B^{\prime}}(v)=\sup _{u \in \overline{\operatorname{Support}(A)}} f_{A^{\prime}}(u)$

- $\sup _{v \in V} f_{B^{\prime}}(v)=\sup _{u \in U} f_{A^{\prime}}(u)$

- If Support $\left(A^{\prime}\right)=\operatorname{Support}(A)$ and $A^{\prime}=m(A)$ then $B^{\prime}=m(B)$

\section{B.6 Kleene-Dienes} by:

With Łukasiewicz's GMP operator conclusions are given

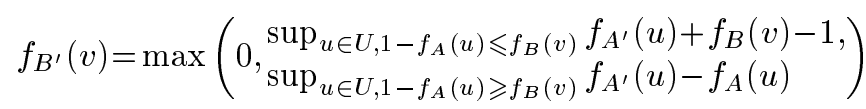

Which gives us:

- $\inf _{v \in V} f_{B^{\prime}}(v)=\sup _{u \in \overline{S u p p o r t}(A)} f_{A^{\prime}}(u)$

\section{B.7 Gödel}

The conclusion of a fuzzy inference given Gödel's implication and the min GMP operator is given by:

$$
f_{B^{\prime}}(v)=\max \left(\sup _{u \in U, f_{B}(v) \geqslant f_{A}(u)} f_{A^{\prime}}(u), f_{B}(v)\right)
$$

Which means:
- $B^{\prime} \supseteq B$

- $\inf _{v \in V} f_{B^{\prime}}(v)=\sup _{u \in \overline{\operatorname{Support}(A)}} f_{A^{\prime}}(u)$

With Lukasiewicz's t-norm we get;

$$
f_{B^{\prime}}(v)=\max \left(\begin{array}{l}
f_{B}(v)+\sup _{u \in U, f_{A}(u) \geqslant f_{B}(v)} f_{A^{\prime}}(u)-1, \\
\sup _{u \in U, f_{A}(u) \leqslant f_{B}(v)} f_{A^{\prime}}(u)
\end{array}\right)
$$

With Goguen's GMP operator we have:

$$
f_{B^{\prime}}(v)=\max \left(\begin{array}{l}
\sup _{u \in U, f_{A}(u) \geqslant f_{B}(v)} f_{A^{\prime}}(u) \times f_{B}(v), \\
\sup _{u \in U, f_{A}(u) \leqslant f_{B}(v)} f_{A^{\prime}}(u)
\end{array}\right)
$$

Which means that for both t-norms we have:

- $\inf _{v \in V} f_{B^{\prime}}(v) \geqslant \sup _{u \in \overline{\operatorname{Support}(A)}} f_{A^{\prime}}(u)$

- If $K \operatorname{ernel}\left(A^{\prime}\right) \cap K \operatorname{Kernel}(A) \neq \varnothing$ then $B^{\prime} \supseteq B$

- Otherwise, if $A^{\prime} \subset A$ and $\operatorname{Kernel}\left(A^{\prime}\right) \cap K \operatorname{Kernel}(A)=\varnothing$ then $\sup _{v \in V} f_{B^{\prime}}(v)=\sup _{u \in U} f_{A^{\prime}}(u)$

\section{B.8 Goguen}

Using Łukasiewicz's t-norm we get the following expression;

$$
\begin{aligned}
f_{B^{\prime}}(v) & =\max \left(\begin{array}{l}
\sup _{u \in U, f_{A}(u) \geqslant f_{B}(v), f_{A}(u)>0} f_{A^{\prime}}(u)+\frac{f_{B}(v)}{f_{A}(u)}-1, \\
\sup _{u \in U, f_{A}(u) \leqslant f_{B}(v)} f_{A^{\prime}}(u)
\end{array}\right) \\
& =\sup _{u \in U, f_{A}(u) \leqslant f_{B}(v)} f_{A^{\prime}}(u)
\end{aligned}
$$

Which implies that: 

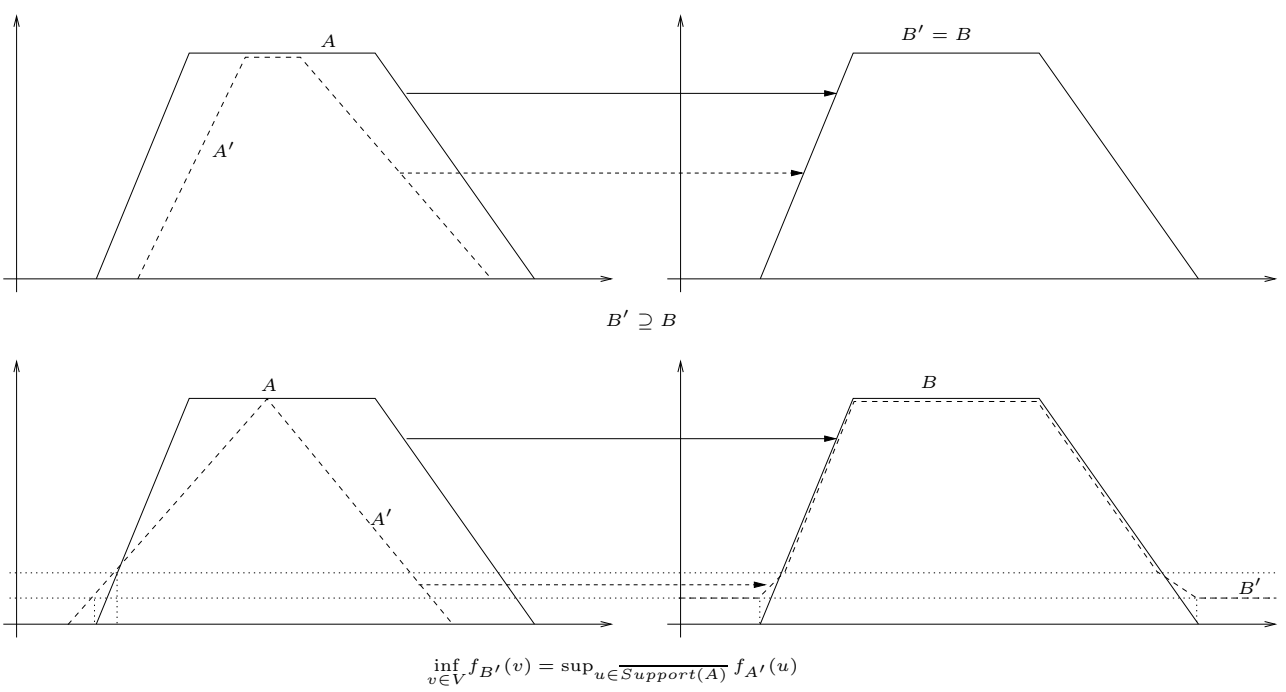

Fig. 2. Essential properties of conclusions with Gödel's implication and Zadeh's t-norm

- $\inf _{v \in V} f_{B^{\prime}}(v) \geqslant \sup _{u \in \overline{\operatorname{Support}(A)}} f_{A^{\prime}}(u)$

- $\sup _{v \in V} f_{B^{\prime}}(v)=\sup _{u \in U} f_{A^{\prime}}(u)$

When combined to Goguen's operator, we have:

$f_{B^{\prime}}(v)=\max \left(\begin{array}{l}f_{B}(v) \times \sup _{u \in U, f_{A}(u) \geqslant f_{B}(v), f_{A}(u)>0} \frac{f_{A^{\prime}}(u)}{f_{A}(u)}, \\ \sup _{u \in U, f_{A}(u) \leqslant f_{B}(v)} f_{A^{\prime}}(u)\end{array}\right)$

Which means:

- $\inf _{v \in V} f_{B^{\prime}}(v) \geqslant \sup _{u \in \overline{\operatorname{Support}(A)}} f_{A^{\prime}}(u)$

- If $\operatorname{Kernel}\left(A^{\prime}\right) \cap K \operatorname{Kernel}(A) \neq \varnothing$ then $B^{\prime} \supseteq B$

- Otherwise, if $A^{\prime} \subset A$ and $K \operatorname{ernel}\left(A^{\prime}\right) \cap K \operatorname{Kernel}(A)=\varnothing$ then $\sup _{v \in V} f_{B^{\prime}}(v)=\sup _{u \in U} f_{A^{\prime}}(u)$

\section{B.9 Łukasiewicz}

The general expression of the conclusion of a fuzzy rule given Łukasiewicz's implication is given by:

$f_{B^{\prime}}(v)=\max \left(\begin{array}{l}f_{B}(v)+\sup _{u \in U, f_{A}(u) \geqslant f_{B}(v)} f_{A^{\prime}}(u)-f_{A}(u), \\ \sup _{u \in U, f_{A}(u) \leqslant f_{B}(v)} f_{A^{\prime}}(u)\end{array}\right)$

From which we see that:

- $\inf _{v \in V} f_{B^{\prime}}(v) \geqslant \sup _{u \in \overline{\operatorname{Support}(A)}} f_{A^{\prime}}(u)$

- If $A^{\prime} \supset A$ then $B^{\prime} \supset B$

- If $A^{\prime} \subset A$ and $\operatorname{Kernel}\left(A^{\prime}\right) \cap \operatorname{Kernel}(A) \neq \varnothing$ then $B^{\prime}=B$

- If $A^{\prime} \subset A$ and $K \operatorname{ernel}\left(A^{\prime}\right) \cap K \operatorname{Kernel}(A)=\varnothing$ then $B^{\prime} \subset B$

Table III reviews the properties we have put forward and groups them with respect to the implications, in order to suggest possible links between implications.

Now, suppose that we have built or learned a fuzzy-rulebase on diseases and their symptoms. Generally speaking, to make a diagnostic a physician usually has to consider only the symptoms. Suppose also that, after the construction of our rules, we observe sufficient data to build symptom-similar cases without diagnostic. If these classes of cases are fuzzy sets defined on the symptom's scale, we now have a way of selecting the fuzzy implication to apply for each rule.

This is what we refer to as 'data-driven' classification of the rules. The semantic interpretation of each rule may, of course, still be that given by Dubois and Prade, but the choice is coherent with the observations. We may well find that different rules, even though they are used in the same context, belong to different classes and should therefore be interpreted differently. For instance, if we were to observe a denormalised class of pain to the lower abdomen, we would have to choose one of the implications exhibiting property 5 to encode the rule linking appendicitis to this particular symptom. Obviously there would still be a choice of sorts, but at least we would know that the implication was an r-implication and thus that the rule was a gradual one.

\section{CONCLUSION}

Our ambition, in this paper as in [8], was, originally, to extend formal fuzzy abductive results to different classes of implications and linguistic modifiers. While working on these results we noticed that the theory contradicted some established results. The explanation of these incoherences lay in the 'impossibility' of observing certain shapes. Yet these shapes did not seem incoherent with the data they were meant to represent. Tracing the incoherence of our results back to the 'observable' shapes of the selected fuzzy implications, we saw that observations were bound by the implication operator. To allow suspected 'data-coherent' observations we needed to find 'deduction-coherent' implications. Available studies of the Generalised Modus Ponens offered information on possible shapes, but did not allow us to definitely rule-out others. Therefore we had to generalise these results to conclude. We would like to ex- 


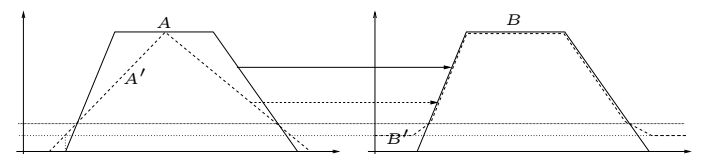

$\inf _{v \in V} f_{B^{\prime}}(v) \geqslant \sup _{u \in \overline{\operatorname{Support}(A)}} f_{A^{\prime}}(u)$

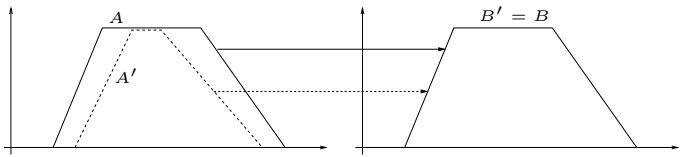

$A^{\prime} \subset A$ and $\operatorname{Kernel}\left(A^{\prime}\right) \cap \operatorname{Kernel}(A) \neq \varnothing$
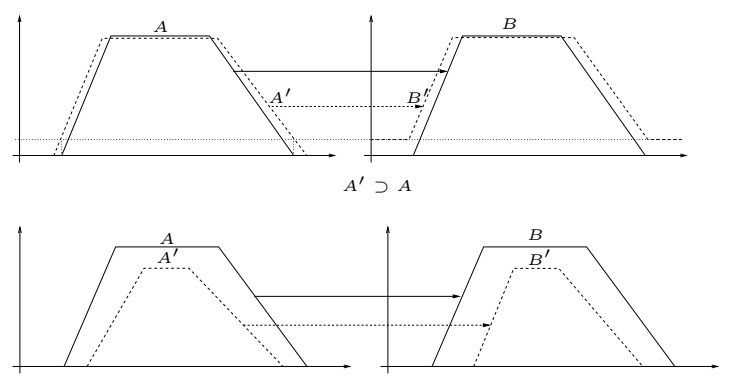

$A^{\prime} \subset A$ and $\operatorname{Kernel}\left(A^{\prime}\right) \cap \operatorname{Kernel}(A)=\varnothing$

Fig. 3. Some properties of GMP conclusions given Lukasiewicz's implication

TABLE III

GENERAL PROPERTIES OF GMP CONCLUSIONS

\begin{tabular}{|c|c|c|}
\hline Property & Implication & t-norm $(\mathrm{s})$ \\
\hline $1 \quad B^{\prime} \equiv B$ & Mamdani & Zadeh \\
\hline $2 \quad B^{\prime} \subseteq B$ & Mamdani & Goguen, Lukasiewicz \\
\hline $3 \quad B^{\prime} \supseteq B$ & Gödel & Zadeh \\
\hline $4 \quad B^{\prime} \supseteq B$ if $\operatorname{Kernel}\left(A^{\prime}\right) \cap \operatorname{Kernel}(A) \neq \varnothing$ & $\begin{array}{l}\text { Gödel } \\
\text { Lukasiewicz }\end{array}$ & $\begin{array}{l}\text { Lukasiewicz } \\
\text { Lukasiewicz }\end{array}$ \\
\hline $5 \quad$ Persistent denormalisation & $\begin{array}{l}\text { Rescher-Gaines } \\
\text { Gödel } \\
\text { Goguen } \\
\text { Lukasiewicz }\end{array}$ & $\begin{array}{l}\text { Zadeh, Goguen, Łukasiewicz } \\
\text { Lukasiewicz } \\
\text { Goguen, Lukasiewicz } \\
\text { Lukasiewicz }\end{array}$ \\
\hline $6 \inf _{v \in V} f_{B^{\prime}}(v)=\sup _{u \in \overline{\operatorname{Support}(A)}} f_{A^{\prime}}(u)$ & $\begin{array}{l}\text { Rescher-Gaines } \\
\text { Kleene-Dienes } \\
\text { Gödel }\end{array}$ & $\begin{array}{l}\text { Zadeh, Goguen, Łukasiewicz } \\
\text { Lukasiewicz } \\
\text { Zadeh }\end{array}$ \\
\hline $7 \quad \inf _{v \in V} f_{B^{\prime}}(v) \geqslant \sup _{u \in \overline{\operatorname{Support}(A)}} f_{A^{\prime}}(u)$ & $\begin{array}{l}\text { Gödel } \\
\text { Goguen } \\
\text { Lukasiewicz }\end{array}$ & $\begin{array}{l}\text { Lukasiewicz } \\
\text { Lukasiewicz } \\
\text { Lukasiewicz }\end{array}$ \\
\hline
\end{tabular}

tend this type of systematic analysis to other implications and their associated GMP operators, or t-norm.

Selecting an implication from the data meant we could interpret our rule-based knowledge using the semantic interpretation of the operators. Our classification of subsets of a rule-base would benefit from interpretations at the implication operator level. Indeed, implications of different types may generate similar shapes. Conversely, some implications of the same type do not accept the same modifications. Therefore, 'observation consistent' implications, which we use to classify our rule-base subsets, may have some semantic proximity and, if not, their differences would entail as many potential interpretations. The properties we have laid out in this comparative study also seem connected to the choice of GMP-operator. This should be taken into account in the semantic interpretation processes.

\section{REFERENCES}

[1] Nédra Mellouli and Bernadette Bouchon-Meunier, "Abductive reasoning and measures of similitude in the presence of fuzzy rules," Fuzzy Sets and Systems 137, pp. 177-188, 2003.

[2] Nédra Mellouli, Le raisonnement abductif flou : théorie et pratique, Ph.D. thesis, LIP6, 122001.

[3] Didier Dubois and Henri Prade, "A typology of 'if... then' rules," in Proceedings of the third International Fuzzy Systems Association Congress, Seattle, 1989, pp. 782-785.

[4] Didier Dubois and Henri Prade, "What are fuzzy rules and how to use them," Fuzzy Sets and Systems, vol. 84, no. 2, pp. 169-189, 1996.

[5] Lotfi A. Zadeh, Information Sciences, vol. 8, chapter The Concept of a Linguistic Variable and its Application to Approximate Reasoning, pp. 301-357, 1975.

[6] Bernadette Bouchon-Meunier and Jia Yao, "Linguistic modifiers and imprecise categories," International Journal of Intelligent Systems, vol. 7, pp. 25-36, 1992.

[7] Sylvie Desprès, Un apport à la conception de systèmes à base de connaissances : Les opérations de déduction floues, Ph.D. thesis, LIP6, 41988.

[8] Adrien Revault d'Allonnes, Herman Akdag, and Bernadette Bouchon-Meunier, "Vers une classification de problèmes abductifs en fonction d'observations possibles," in Actes des Rencontres Francophones sur la Logique Floue et ses Applications (LFA'2006), 2006, pp. 21-28. 